\title{
The Multi-dimensional Langevin Approach to the Description of Fusion-fission Reactions
}

\author{
G. I. Kosenko, ${ }^{*, a}$ F. A. Ivanyuk, ${ }^{b}$ and V. V. Pashkevich ${ }^{\mathrm{c}}$ \\ ${ }^{a}$ Omsk State University, 644077 Omsk, Russia \\ ${ }^{\mathrm{b}}$ Institute for Nuclear Research, 03028 Kiev-28, Ukraine \\ ${ }^{c}$ Laboratory for Theoretical Physics, JINR, 141890 Dubna, Russia
}

Received: November 13, 2001; In Final Form: April 25, 2002

\begin{abstract}
The two stage approach to the description of fusion-fission reactions is suggested. On each stage (fusion or fission) the tree-dimensional Langevin equation for the variables describing the shape of nuclear system is solved. The results obtained on the first stage are used as the input data for description of fission dynamics. In this way it turned out possible to describe for the reaction ${ }^{18} \mathrm{O}+{ }^{208} \mathrm{~Pb}$ simultaneously both fusion and fission cross sections, the energy and mass distribution of fission fragments, the probability of the evaporation residue formation, the dependence of pre-fission neutron multiplicities on the fragment mass number. From the results of the computations it follows that the observed quantities of the fission process can be reproduced without formation of compound nucleus. At the same time the duration of the process is so large that events of quasi-fission and "true" fission of compound nucleus can not be distinguished.
\end{abstract}

\section{Introduction}

For many years the fusion and fission processes were studied separately. This was mainly due to the fact that the relatively light ions were used in fusion reaction and it was assumed that the ions are absorbed very fast by the target nucleus. With the increasing weight of the ions the picture changes considerably. ${ }^{1}$ Now, when the Uranium ions are used as the projectile the description of fission independently from the fusion stage is not justified.

One of the examples of such approach is the concept of dinuclear system (DNS). ${ }^{2-5}$ In this model it is assumed that after the touching point both target and projectile keep their "individuality" - spherical shape and shell structure. The entrance channel in this model is taken into account through by the probability of the formation of DNS which is calculated on the basis of optical model. This is the only memory about entrance channel. So the initial stage and the further evolution of the system is carried out within very different approaches.

More consistent are the approaches developed in References 6,7 . The parameters of the entrance channel (the dependence of the compound nucleus formation probability on the angular moment $)^{8}$ and the further evolution of the composite system ${ }^{6}$ are considered within the same approach. However whereas the DNS-approach starts from the contact of two nuclei in References 6,7 this moment is missing completely. Once the probability of compound nucleus is known, the evolution of the fissioning system starts in References 6,7 from the ground state. Thus the "pre-history" is ignored completely. On this way the considerable part of the information on systems evolution is lost.

In present work an attempt is undertaken to consider both the approaching phase and the fission within the same framework, namely by solving the Langevin equations for the variables which specify the shape of composite system. As an example we will consider the reaction ${ }^{18} \mathrm{O}+{ }^{208} \mathrm{~Pb}$ for which the detailed experimental information is available. Note also that both nuclei are spherical.

\section{The Model}

The description of the fusion-fission reactions will be carried out in two stages. Both stages are described by threedimensional Langevin equation. On the first stage the approaching phase is considered. The information obtained on this step

*Corresponding author. E-mail:kosenko@nrsun.jinr.ru. is used as the input data for describing the further evolution of the composite system. Unfortunately, at present there is no shape parametrization which would describe well both divided and compact shapes. Thus on both stages different parametrization will be used. On each stage the evolution of large number of collisions is considered. Each of them would be referred as "trajectory" in the space of deformation parameters.

2.1. Equations of Motion. To describe the dynamics of the fusion-fission reaction we will use the Langevin equations. For the fusion stage we will solve the equations ${ }^{9}$ :

$$
\begin{aligned}
\frac{d r}{d t} & =\frac{p_{r}}{m}, \\
\frac{d p_{r}}{d t} & =-\frac{\partial V(r, \vec{\alpha})}{\partial r}-\frac{L^{2}}{m r^{3}}-K_{r}^{r} \frac{p_{r}}{m}-\sum_{j} K_{r}^{j} \frac{\pi_{j}}{D_{j}}+\xi \\
\frac{d \alpha_{i}}{d t} & =\frac{\pi_{i}}{D_{i}}, \\
\frac{d \pi_{i}}{d t} & =-\frac{\partial V(r, \vec{\alpha})}{\partial \alpha_{i}}-K_{i}^{r} \frac{p_{r}}{m}-\sum_{j} K_{i}^{j} \frac{\pi_{j}}{D_{j}}-C_{i} \alpha_{i}-\frac{1}{2} \xi .
\end{aligned}
$$

Here $r$ is the distance between centers of mass of colliding nuclei, $p_{r}-$ conjugated momentum, $m-$ the reduced mass, $V(r, \vec{\alpha})$ - the interaction energy. The latter includes the Coulomb and nuclear parts. The nuclear part $V_{G K}$ was calculated according to Gross and Kalinowski, ${ }^{12}$ the $K_{i}^{j}$ is the tensor of friction parameters. The angular momentum $L$ is expressed in units $\hbar$. The second couple of eq 1 describes the shape evolution of colliding nuclei. The quantities $\alpha_{i}$ and $\pi_{i}$ are the collective variables describing the shape of nucleus and conjugated momentum correspondingly. The index $i$ attains the values "target" or "projectile", the $D_{i}$ and $C_{i}$ are the friction and mass parameters with respect to $\alpha_{i}$. Both $D_{i}$ and $C_{i}$ were defined within liquid drop model. The quantity $\xi$ describes the random force acting upon the system and leading to the fluctuations. The magnitude of the fluctuations depends on the excitation energy of the system, so at initial moment it is equal to zero. During the approach the part of the kinetic energy turns into heat due to the friction in the system. Consequently the magnitude of fluctuations gets different from zero and increases with time.

The system of eq 1 does not include the equation for angular momentum $L$ of the system. It was assumed to be a constant for each trajectory.

For the mono system the Langevin equations are of the form $^{13}$ :

$$
\frac{d q_{i}}{d t}=M_{i j} p_{j}
$$




$$
\frac{d p_{i}}{d t}=-\frac{\partial V(\vec{q})}{\partial q_{i}}-\frac{1}{2} p_{j} p_{k} \frac{\partial \mu_{j k}}{\partial q_{i}}-\gamma_{i j} M_{j k} p_{k}+\xi_{i}
$$

where $q_{i}$ are the collective variables fixing the shape of mono system and $p_{i}$ are the conjugated momenta. The $\gamma_{j k}$ and $\mu_{j k}=$ $\left\|m_{j k}\right\|^{-1}$ are the friction and mass parameters. For the compact shapes these parameters were computed within the linear response approach. ${ }^{16,17}$ The explicit expressions and numerical results for $\gamma_{j k}$ and $m_{j k}$ can be found in Reference 18.

2.2. Collective Co-ordinates. On the approach phase the projectile and target get deformed both by Coulomb and nuclear interaction in order to reduce the interaction energy. So, one should account for the deformation of the projectile and target. In the present work we will take into account only the most important quadrupole deformation. I.e., the shape of nuclear surface is parametrized by

$$
R_{i}(x)=\lambda_{i}^{-1} R_{0}^{i}\left(1+\alpha_{2}^{i} P_{2}(x)\right) .
$$

Here $R_{0}$ is the radius of the sphere with equal volume, index $i$ denote "target" or "projectile", the parameter $\alpha_{2}^{i}$ defines the deformation of the projectile or the target, $\lambda_{i}$ is the normalization factor (defined from the volume conservation condition). It is assumed that on the approaching stage the symmetry axis of projectile and target coincide (Figure 1), i.e. the rotation of ions is not taken into account. This approximation seems quite reasonable, though it may lead to some reduction of the fusion cross section. ${ }^{10}$

On the second stage, when the projectile and target formed a bound system, its shape is parametrized in terms of Cassini ovaloids, ${ }^{11}$ i.e. by the profile function $\rho_{s}(z)$ which is given parametrically as

$$
\begin{aligned}
\rho_{s}(x)= & \frac{1}{\sqrt{2}}\left[\left(R^{4}+2 s R^{2}\left(2 x^{2}-1\right)+s^{2}\right)^{1 / 2}\right. \\
& \left.-R^{2}\left(2 x^{2}-1\right)-s\right]^{1 / 2}, \\
z(x)= & \frac{\operatorname{sign}(x)}{\sqrt{2}}\left[\left(R^{4}+2 s R^{2}\left(2 x^{2}-1\right)+s^{2}\right)^{1 / 2}\right. \\
& \left.+R^{2}\left(2 x^{2}-1\right)+s\right]^{1 / 2} \\
R(x)= & \lambda^{-1} R_{0}\left(1+\sum_{m} \alpha_{m} P_{m}(x)\right) \\
s= & \varepsilon R_{0}^{2} .
\end{aligned}
$$

Here $s$ is the square of distance from the focus of Cassini ovals to the center of co-ordinate system. The surface of the (axially symmetric) nucleus is obtained by the rotation of the profile function $\rho_{s}(z)$ around $z$-axis. The shape of the surface is fixed by the deformation parameters $\varepsilon$ and $\alpha_{m}$. Near the scission it is convenient to use instead of $\varepsilon$ as an independent variable the parameter $\alpha$ which relates to $\left(\varepsilon, \alpha_{m}\right)$ as

$$
\begin{aligned}
\varepsilon= & \frac{1}{4}(\alpha-1)\left[\left(1+\sum_{m=1,4} \alpha_{m}\right)^{2}+\left(1+\sum_{m=1,4}(-1)^{m} \alpha_{m}\right)^{2}\right] \\
& +\frac{1}{2}(\alpha+1)\left[1+\sum_{m=1,4}(-1)^{m} \alpha_{2 m}(2 m-1) ! ! /\left(2^{m} m !\right)\right]^{2}
\end{aligned}
$$

The advantage of parameter $\alpha$ is due to the fact that for a shape with vanishing neck $\alpha=1$ for any value of $\alpha_{m}$. So, $\alpha=0$ corresponds to spherical shape and $\alpha=1$ correspond to the shape with zero neck.

When we switch from two separated ions to a bound system, we conserve the full elongation and the mass asymmetry of the system. Namely, the ratio of the volumes of left and right part of the system (which are divided the zero neck) are the same as the one for colliding ions. And the sum of radiuses of ions along the symmetry axis is equal to the elongation of the whole

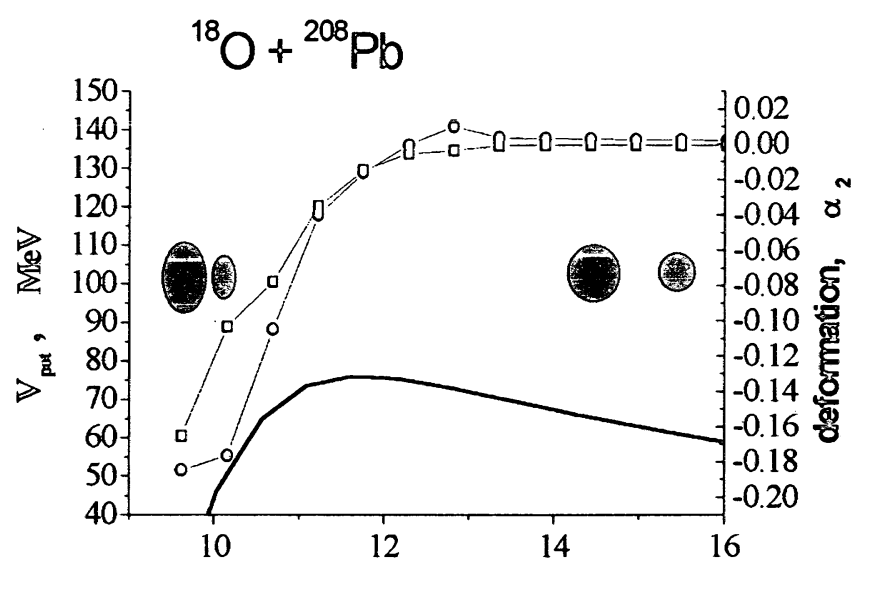

relative distance, fim

Figure 1. The dependence of the potential energy (solid curve, left axis) and the mean deformations $\alpha_{2}$ of the target (squares) and projectile (circles, right axis) versus the distance $r$ between the projectile and target. The shapes of the ions at the initial point (right) and near the touching point (left) are also shown.

system. The parameters of the compact system were defined numerically for each trajectory.

In the present work we use three deformation parameters both for bound and separated shapes. In the first case these are the distance $r$ between the centers of mass of target and projectile and the parameters $\alpha_{i}$ of their (quadrupole) deformation. In the second case - the deformation parameters $\alpha, \alpha_{1}$, and $\alpha_{4}$ which fix the total elongation of the system, mass asymmetry, and thickness of the neck.

2.3. The Potential Energy. The potential energy includes the Coulomb, rotational, and nuclear parts. Below only the last part is shown. The nuclear interaction of two ions in the entrance channel was described by means of Gross-Kalinowski potential $V_{G K}{ }^{12}$

$$
\begin{aligned}
V_{G K} & =\frac{1}{2}\left(V_{12}+V_{21}\right) \\
V_{12} & =\int V_{1}\left(\vec{r}-\vec{r}^{\prime}, \alpha_{1}\right) \rho_{2}\left(\vec{r}^{\prime}, \alpha_{2}\right) d \vec{r}^{\prime} \\
V_{i}\left(\vec{r}, \alpha_{i}\right) & =\frac{V_{p}}{1+\exp \left(\frac{r-R_{p}\left(\alpha_{i}, x\right)}{a_{p}}\right)} \\
\rho_{i}\left(\vec{r}, \alpha_{i}\right) & =\frac{\rho_{0}}{1+\exp \left(\frac{r-R_{d}\left(\alpha_{i}, x\right)}{a_{d}}\right)} \\
R_{p}\left(\alpha_{i}, x\right) & =1.25 A_{i}^{1 / 3}\left(1+\alpha_{i} P_{2}(x)\right)[\mathrm{fm}], \\
R_{d}\left(\alpha_{i}, x\right) & =\left(1+\alpha_{i} P_{2}(x)\right)\left(1.12 A_{i}^{1 / 3}-0.86 A_{i}^{-1 / 3}\right)[\mathrm{fm}]
\end{aligned}
$$

with $\rho_{0}=0.17 \mathrm{fm}^{-3}, a_{d}=0.54 \mathrm{fm}$. The parameters of the potential $V_{p}$ and $a_{p}$ were chosen according to Reference 14 . In the calculations the mash $100 \times 41 \times 41$ in the deformation space was used. The $r$-dependence of the potential energy for $L=0$ is shown in Figure 1.

The potential energy for the compact shape was computed by the shell correction method ${ }^{20}$ as the sum of liquid-drop part and the shell correction. The damping of the shell correction with growing excitation energy was taken into account according to Reference 15.

Figure 2 shows the map of the potential energy in $\alpha, \alpha_{1}$ coordinates (the parameter $\alpha_{4}$ which defines the thickness of the neck is fixed equal to -0.18). One can see that with growing temperature the potential energy gets more smooth and more close to the liquid-drop deformation energy. The two fission valley merge into one. Thus, depending on the excitation energy, the mass distribution of the fission fragments could be symmetric or not. 


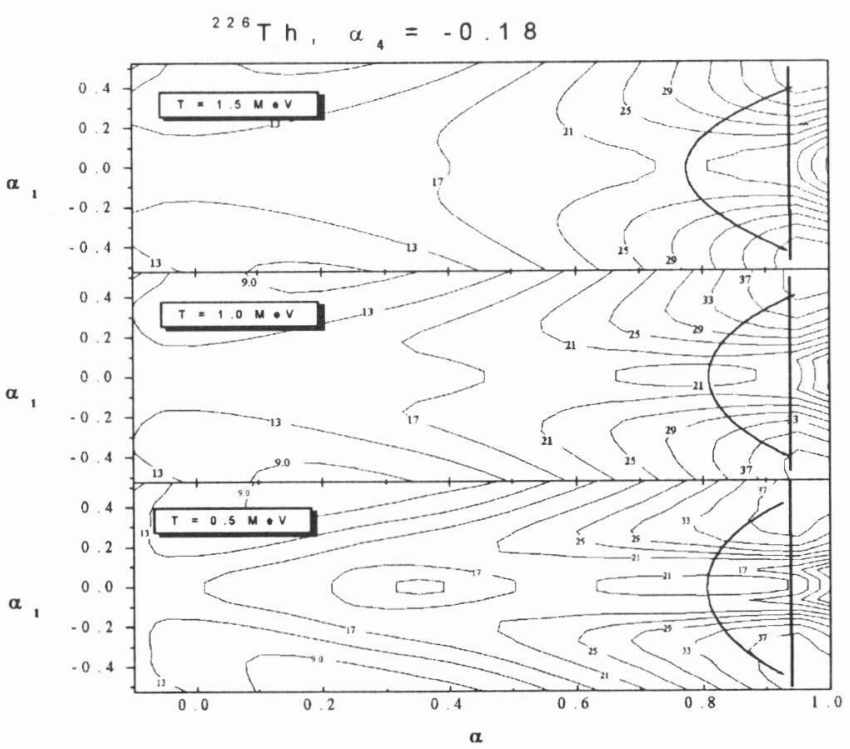

Figure 2. The potential energy of ${ }^{226} \mathrm{Th}$ in $\alpha, \alpha_{1}$ co-ordinates for $\alpha_{4}=$ -0.18 . The contour maps are given for three values of the temperature $T$. The vertical line marks the value of $\alpha_{1}$ at which the stability of the nucleus with respect to scission is lost.

\section{The Entrance Phase}

The potential energy of spherical colliding ions is shown in Figure 1 by solid line. Of course, the potential energy would change due to the deformation of ions. The mean deformations of projectile (circles) and target (squares) are shown in Figure 1 too. The value of deformation is indicated on the right $y$-axis. One can see that far away from the barrier parameter of deformation $\alpha_{2}$ is zero or very slightly positive. Near the barrier $\alpha_{2}$ gets negative, i.e. both projectile and target get oblate. This lead to the increase of Coulomb interaction. The smaller is distance $r$ between centers of mass the larger is the deformation. Contrary to the case of spherical nuclei, the touching of deformed surfaces practically can not be reached. The evolution of a trajectory in entrance channel was stopped when the distance between the surfaces of ions was smaller than half of the sum of the diffuseness parameters $a_{p}$ (see eq 5) of projectile and target. The cross section obtained in this way was identified with the touching cross section. This values are shown by open triangles in Figure 4.

By solving eq 1 we obtain the information on the entrance channel: the probability of the touching, the distribution of the excitation energy $E_{\text {int }}^{*}$ at the touching point and deformations of the fragments at the touching point. These distributions are shown in Figure 3. The top panel shows the distribution of the intrinsic excitation energy $E_{\text {int }}^{*}$ at the touching point on the initial angular momentum for the initial energy $E_{\text {lab }}=90 \mathrm{MeV}$. One can see that for each angular momentum the excitation energy is distributed around some mean value. With growing angular momentum, the mean value of the excitation energy goes to zero due to the redistribution of the initial energy between intrinsic excitation and rotation.

The middle panel of Figure 3 shows the probability to reach the touching point as the function of initial angular momentum. The open squares correspond to the initial energy $90 \mathrm{MeV}$, the filled triangles - to the energy $78 \mathrm{MeV}$. In both cases, the probability decreases from its maximal value to zero quite smoothly due to the presence of random force in eq 1 . The absolute value of the probability for these two cases differs very much from each other. In the second case, when $E_{\text {lab }}=78 \mathrm{MeV}$ the probability to reach the touching point is very small even for head-on collisions. In this case $E_{\text {lab }}$ energy is almost equal to the fusion barrier.

The bottom panel shows the distribution of the deformation of the ions at the touching point. The projectile deformation

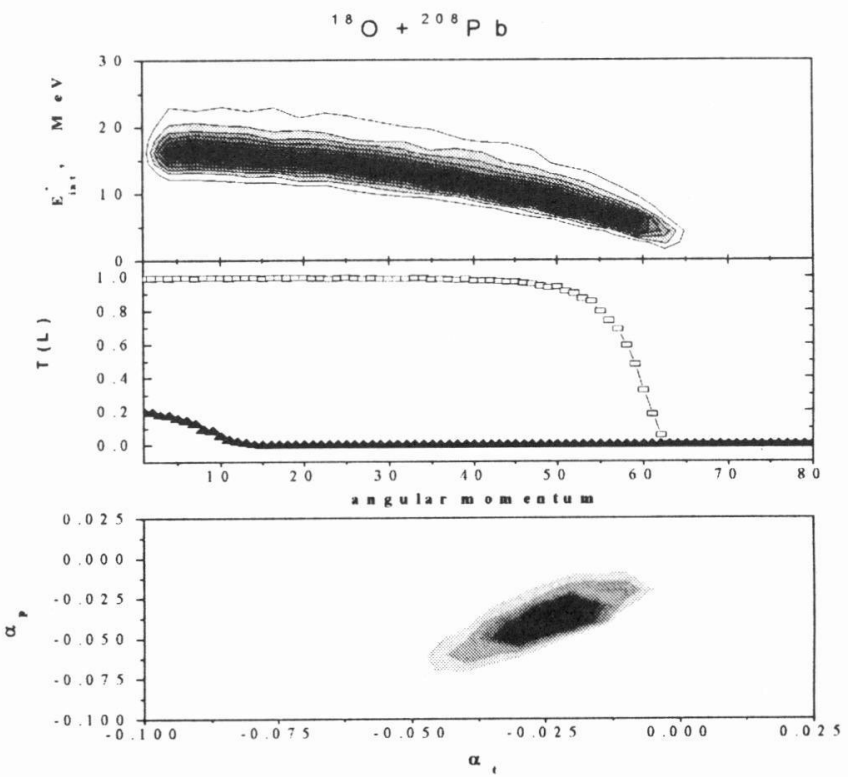

Figure 3. The distribution of the excitation energy (top) of the fragments at the touching, and the touching probability (middle) versus initial angular momentum; bottom - the deformations of the projectile and target at the touching point.

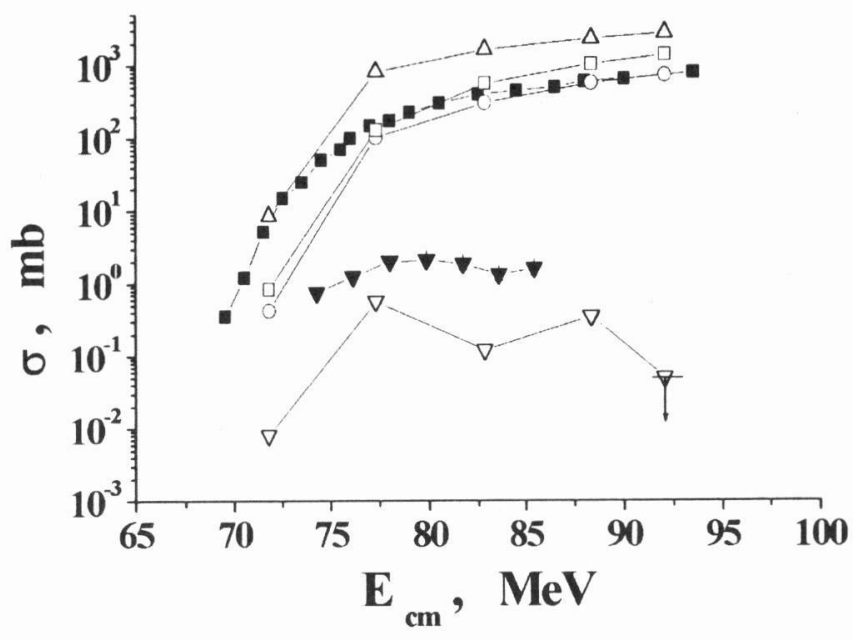

Figure 4. The dependence of the touching cross section (open uptriangles), fusion (open squares) and fission (open circles) cross sections on the $c . m$. energy of the colliding ions. The open down-triangles mark the cross sections of the trajectories which did not scission during $500 \times 10^{-21} \mathrm{~s}$. The filled squares and triangles are the experimental values of the fusion ${ }^{21}$ and evaporation residue cross sections for the reaction ${ }^{16} \mathrm{O}+{ }^{208} \mathrm{~Pb}$.

is indicated on the $y$-axis, and deformation of the target - on the $x$-axis. The negative values of $\alpha_{2}$ correspond to the oblate shapes. The mean value of deformation was shown in Figure 1 as a function of the distance $r$ between the target and projectile.

\section{The Evolution of Composite System}

First of all, we have to define the initial shape of the mono system. The $\alpha$ parameter was put equal to 1 , which corresponds to touching configuration with zero neck. Then for each trajectory we fix by the Neimann ("hit and miss") method the deformations of the colliding ions, see bottom of Figure 3. These two deformations define the elongation of the whole system and the target to projectile mass ratio defines the asymmetry of the whole system. For given elongation and asymmetry we found numerically the values of the asymmetry parameter $\alpha_{1}$ and the neck parameter $\alpha_{4}$. After that, we used the same procedure and distribution from the top part of Figure 3 and defined of the excitation energy $E_{\mathrm{int}}^{*}$ and angular momentum of the mono system.

All this information was used as input data for calculation of the evolution of the mono system, eq 2 . For given $\alpha, \alpha_{1}$, and 
$\alpha_{4}$, the Coulomb, surface, and rotational energy of the mono system was calculated (the rigid body moment of inertia was used). The initial excitation energy defines the temperature of the system and, consequently, the damping of the shell effects. Besides, we assumed that the collective kinetic energy is given by the relation

$$
E_{\mathrm{kin}}=E_{\mathrm{tot}}-V_{\mathrm{pot}}-E_{\mathrm{int}}^{*} .
$$

Here $E_{\mathrm{tot}}$ is the total energy defined from the initial kinetic energy of ions and reaction $Q$ value, $V_{\mathrm{pot}}$ - potential energy for given collective variables and temperature.

The eq 2 were solved numerically. From the solution of eq 2 it follows that the system can break apart practically at once or "travel" for some time on the surface shown in Figure 2. This "travel" can lead either to fusion or fission. The computed fusion and fission cross sections are shown in Figure 4 together with experimental values for the fusion cross section. One can see that computed and experimental values of fusion cross section are close to each other only for high energy region. When the energy of colliding ions is close to the fusion barrier, one should take into account of the tunneling possibility through the barrier. In the present work such possibility was not taken into account since the approach developed in Reference 9 and used here considers only processes with the energy above a fusion barrier.

Now we would like to discuss the problem which events should be considered as true fission and which - as quasifission. The results presented in Figure 4 were obtained in the following way. The whole region of deformation of compact system was divided into three parts: the first part is the region between the shape with zero neck (touching point, $\alpha=1.0$ ) and vertical line $\alpha \sim 0.94$ shown in Figure 2. This line shows the deformation where the liquid drop loses its stability with respect to scission. ${ }^{19}$ I.e., the system moving from the ground state separates into two fragments as soon as it crosses the line $\alpha \sim 0.94$. For the motion in opposite direction from $\alpha=1.0$ the system becomes stable to scission as soon as it crosses the line $\alpha \sim 0.94$. So, in our considerations, all the trajectories which cross the line $\alpha \sim 0.94$ give contribution to the fusion cross section. If the system scissions before $\alpha=0.94$, such event would correspond to deep inelastic collisions which are of no interest for the present.

The second region of interest is $0.83 \leq \alpha \leq 0.94$. The value $\alpha=0.83$ corresponds to the fission barrier (for symmetrical shape). So, if the trajectory crosses the line $\alpha=0.83$, such events are considered as the fission events. The other trajectories which go back before $\alpha=0.83$ are quasi-fission or fast-fission events.

Why are trajectories with minimal parameter $\alpha=0.83$ the fission trajectories? Let us look at Figure 5. The top panel shows the two time distributions of the decay events. The solid curve presents the decay time distribution for all trajectories taken into account. The curve with symbols shows the distribution of events with minimal deformation less that $\alpha=0.83$. One can see that all events with very short evolution time are excluded. The evolution time for this events is shorter than $10 \times 10^{-21} \mathrm{~s}$. But it is the time of reaction of the deep inelastic collision. So, we get the events with a long time evolution. The fission events are the same events. At the same time, the events longer than $150 \times 10^{-21} \mathrm{~s}$ are practically absent. Such events can be considered as contributing to the evaporation residue formation. The cross section of these events is shown by open down-triangles in Figure 4. We would consider these data as an upper estimate for the evaporation residue cross section.

Another confirmation that the events with the evolution time longer then $150 \times 10^{-21} \mathrm{~s}$ are the fission events, could be found looking at the mass-energy distribution of the fragments. In the distribution shown in the middle part of Figure 5 all the trajectories are taken into account. The distribution shown in the lower part includes only the trajectories with minimal deforma-
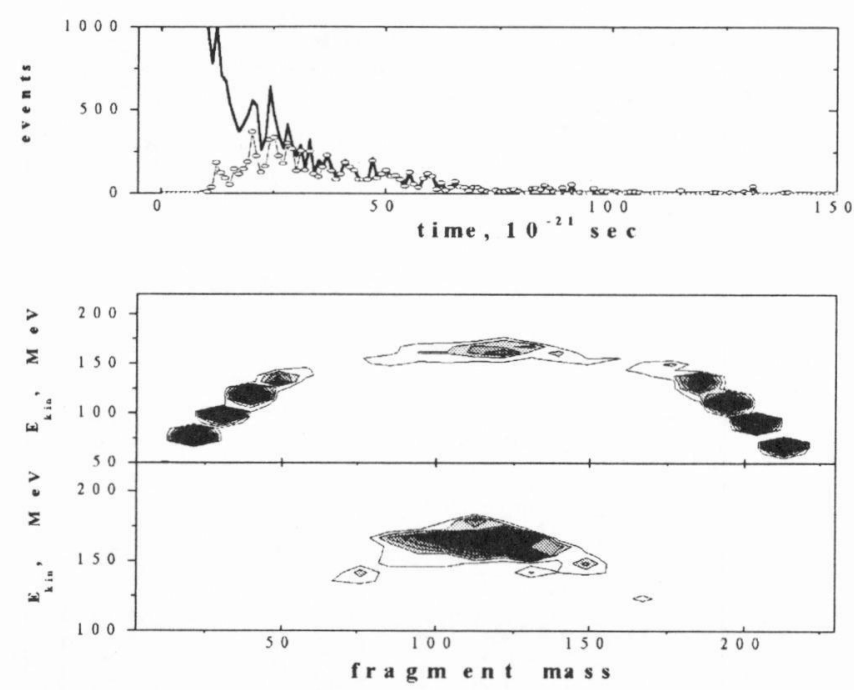

Figure 5. Upper panel: The distribution of decay events on the reaction time. Solid line - all trajectories are taken into account, line with symbols - the contribution from trajectories with minimal deformation less than $\alpha=0.83$, the fission trajectories. Lower panels: Mass-energy distribution of the fragments. Middle panel — all trajectories are taken into account. Bottom - the contribution from trajectories with minimal deformation less than $\alpha=0.83$, the fission trajectories.

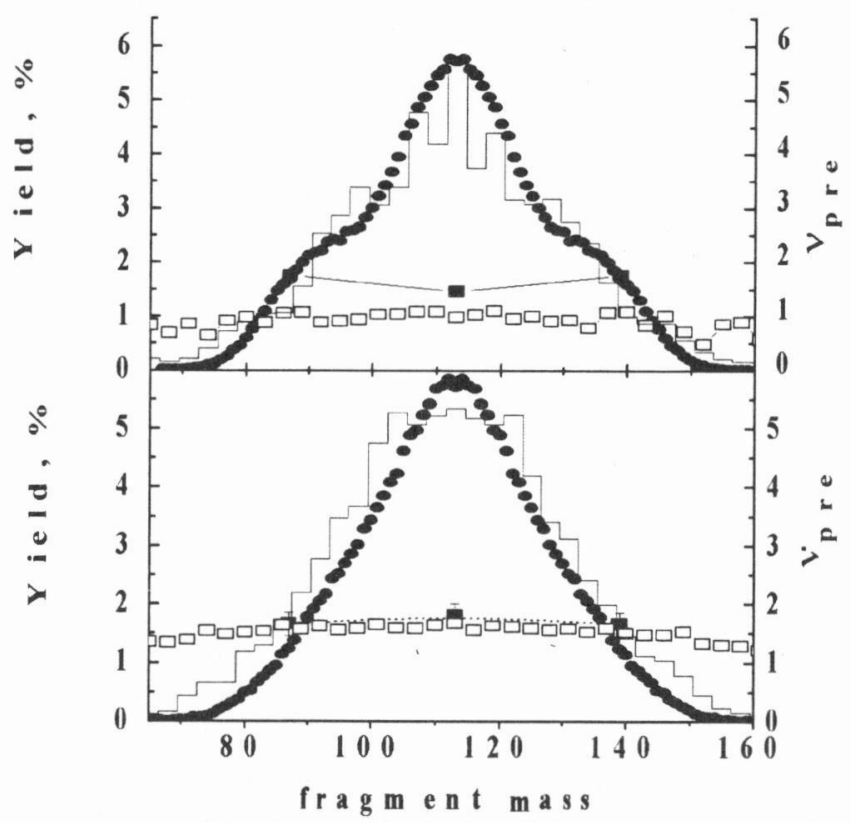

Figure 6. The distribution of the fission fragments and neutron multiplicity on the fragment mass. The initial energy of ions is $78 \mathrm{MeV}$ (top) and $90 \mathrm{MeV}$ (bottom). The left y-axis — fragments yield, right y-axis neutron yield. The heavy dots (the fragment mass distribution) and line with the full squares (neutrons) show the experimental values. ${ }^{23}$ The histogram and line with the open squares show the calculated values, accordingly of the fragment mass distribution and of the neutrons.

tion smaller than $\alpha=0.83$. We can see that the lower part of Figure 5 differ from the middle one drastically. All the events with large asymmetry of fragments disappeared, because the short time is not enough for deep changes of the nuclear structure. Only the central part of distribution have survived when we exclude a short time events. Note that the exactly such type of distribution is observed in the fission experiments. ${ }^{22}$

One more argument could be given by the mass distribution of fission fragments and corresponding neutron multiplicities, see Figure 6. Here we include the trajectories with evolution time larger than $10 \times 10^{-21} \mathrm{~s}$ or only the trajectories shown at the bottom part of Figure 5. We can see that for the energy $E_{\text {lab }}=90 \mathrm{MeV}$, the computed results reproduce rather well the experimental data for the mass distribution and neutron multiplicity. For smaller energy $E_{\text {lab }}=78 \mathrm{MeV}$ the agreement is not so good. The energy $E_{\text {lab }}=78 \mathrm{MeV}$ is practically equal to fusion 


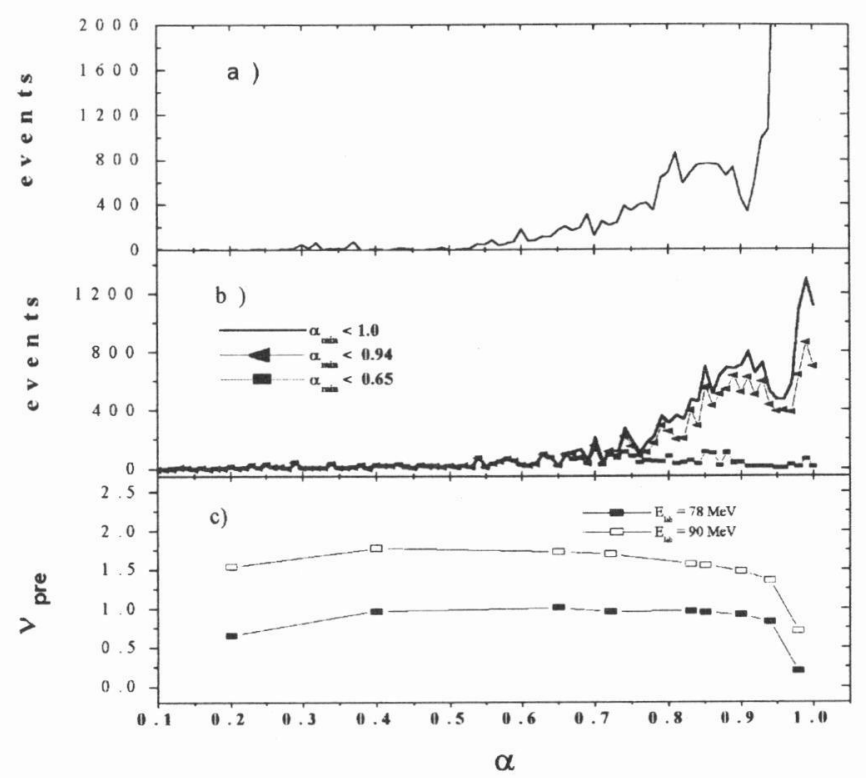

Figure 7. The distribution of decay events with respect to the smallest reached deformation. Top - the decay events. Middle - the neutrons evaporated from all trajectories (solid line), from the trajectories with deformation smaller than $\alpha=0.94$ (line with triangles), and from the trajectories with deformation smaller than $\alpha=0.64$ (line with squares). Bottom - the mean number of evaporated neutrons per decay.

barrier in the entrance channel. As it was mentioned already, the computed touching cross section in this case is smaller than the experimental value, see Figure 4 . Very likely, for this energy one should account for the tunneling through the barrier. Nevertheless, the mass distribution is reproduced rather well.

In case $E_{\text {lab }}=78 \mathrm{MeV}$, the excitation energy is very small and shell effects lead to the "shoulders" in the mass distribution corresponding to mass asymmetric fission. One can see that the "shoulders" are reproduced by the computations. The computed neutron multiplicity is somewhat smaller than the experimental value. There is even some disagreement in the dependence of neutron multiplicity on the mass of fission fragment. Whereas experimental values show some minimum for mass symmetric fission, the computed results are rather independent of the fragment mass.

Figure 7 shows the dependence of the decay events and the number of evaporated neutrons at the smallest deformation reached by trajectory. The panel (a) shows the distribution of all decay events. This distribution has a main bump in the region of $\alpha \sim 1$ (short time events or of the deep inelastic events). The position of the second bump is in barrier region. Many trajectories can not cross the barrier. The main part of the fission trajectories returns from barrier region and minor part of the all trajectories crosses the barrier and go to ground state.

The panel (b) shows of distribution of the positions where the neutrons were evaporated. The solid line include the neutrons evaporated from all trajectories, naturally that this distribution is very similar to the distribution of the panel (a). The line with triangles shows the neutrons evaporated from trajectories with minimal deformation smaller than $\alpha=0.94$, in other words these are neutrons from the fusion trajectories. And the line with squares presents the neutrons evaporated from "pure" fission trajectories, with minimal deformation smaller than $\alpha=0.64$. This distribution is very close to a uniform one. It means that the trajectories on which a lot of neutrons are evaporated before the barrier can not cross it. This conclusion is supported by the panel (c). Here we show the number of the neutrons per decay events versus the position of the minimal deformation. In other words, this is the ratio of the data from the middle and top panels (all trajectories are included). The black symbols correspond to the energy $E_{\text {lab }}=78 \mathrm{MeV}$ and the open symbols - to energy $E_{\text {lab }}=90 \mathrm{MeV}$.

\section{Summary}

We have computed the fusion and fission cross section within the approach based on Langevin equation both for entrance and fission channels. The evaporation residue cross section is also estimated. The mass-energy distributions of the fragments and the dependence of neutron multiplicities on the fragment mass is obtained. The computed results are in good agreement with the experimental data.

The analysis of the numerical results allows us to make the conclusion that the classical compound nucleus is not formed during the collision. At the same time, the distribution of fragments is the same as for the fission of compound nucleus. Moreover, the main part of trajectories does not reach the deformations smaller than the barrier and the main part of neutrons are evaporated around the barrier. In the result of neutron evaporation, the system loses considerable part of kinetic energy, and can not overcome the barrier.

Thus, in order to reach the ground state, the system should have enough energy above the Coulomb barrier. In the synthesis of superheavy elements, these experiments were successful in which system evaporated 2-3 neutrons before it reached ground state. $^{24}$

The authors realize that the results are obtained for one concrete reaction and other fusion-fission reaction should be described. Also the agreement with the experiment for near the barrier energy is not very good. The investigations in this directions would be the subject of future studies.

Acknowledgements. The authors would like to express their gratitude to Yu. Ts. Oganessian, M. G. Itkis, Y. Abe, S. Yamaji, A. Ya. Rusanov, V. S. Salamatin, D. V. Vanin, A. D. Adeev, Y. Aritomo, T. Wada, R. S. Kurmanov, A. A. Goverdovsky, and F. Hanappe for helpful discussions. One of us (G.I.K) thanks the administration of Flerov Laboratory, JINR, Dubna, RIKEN, Tokyo, and Institute for Theoretical Physics, Kyoto for hospitality during his work at these institutes. The work was partly supported by INTAS grant No.11929.

\section{References}

(1) V. V. Volkov, Nuclear Deep Inelastic Reactions (Energoatomizdat, Moscow, 1982).

(2) N. V. Antonenko, E. A. Cherepanov, A. K. Nasirov, V. P. Permjakov, and V. V. Volkov, Phys. Lett. B 319, 425 (1993).

(3) N. V. Antonenko, R. V. Jolos, G. G. Adamian, and A. K. Nasirov, Phys. Part. Nucl. 25, 583 (1994).

(4) G. G. Adamian, N. V. Antonenko, and R. V. Jolos, Nucl. Phys. A 584, 205 (1995).

(5) A. Diaz-Torres, G. G. Adamian, N. V. Antonenko, and W. Scheid, Phys. Rev. C 64, 024604 (2001).

(6) I. I. Gonchar, Phys. Part. Nucl. 26, 394 (1995).

(7) K. Pomorski, B. Nerlo-Pomorska, A. Surowiec, M. Kowal, J. Bartel, K. Dietrich, J. Richert, C. Schmitt, B. Benoit, E. de Goes Brennand, L. Donadille, and C. Badimon, Nucl. Phys. A 679, 25 (2000).

(8) P. Fröbrich and J. Marten, Nucl. Phys. A 545, 854 (1992).

(9) P. Fröbrich and S. Y. Xu, Nucl. Phys. A 477, 143 (1988).

(10) P. Fröbrich and G. Grawert, Nucl. Phys. A 451, 338 (1986).

(11) V. V. Pashkevich, Nucl. Phys. A 169, 275 (1971); 477, 1 (1988).

(12) D. H. E. Gross and H.Kalinowski, Phys. Rep. 45, 175 (1978).

(13) Y. Abe, C. Gregoire, and H. Delagrange, J. Phys. (Paris) 47, c329 (1986).

(14) P. Fröbrich, Phys. Rep. 116, 337 (1984).

(15) A. V. Ignatyuk, G. N. Smirenkin, and A. S. Tishin, Yad. Fiz. 21, 485 (1975).

(16) H. Hofmann, Phys. Rep. 284, 137 (1997). 
(17) F. A. Ivanyuk, H. Hofmann, V. V. Pashkevich, and S. Yamaji, Phys. Rev. C 55, 1730 (1997).

(18) F. A. Ivanyuk, Proceedings of the International Conference on Nuclear Physics "Nuclear Shells - 50", Dubna, Russia, Apr. 21-24, 1999 (World Scientific, Singapore, 2000), p. 456.

(19) U. Brosa, S. Grossmann, and A. Müller, Phys. Rep. 197, 167 (1990).

(20) J. Damgaard, H. C. Pauli, V. V. Pashkevich, and V. M. Strutinsky, Nucl. Phys. A 135, 432 (1969).

(21) E. Vulgaris, L. Grodzins, S. G. Steadman, and R. Ledoux, Phys. Rev. C 33, 2017 (1986).

(22) M. G. Itkis and A. Ya. Rusanov, Phys. Part. Nucl. 29, 160 (1998).

(23) I. V. Pokrovski, M. G. Itkis, I. M. Itkis, E. M. Kozulin, N. A. Kondratiev, Yu. Ts. Oganessian, E. V. Prokhorova, A. Ya. Rusanov, S. P. Tretiakova, A. Kelic, G. Rudolf, L. Stuttge, and F. L. Calabretta, Proceedings of the International Conference on Nuclear Physics "Nuclear Shells - 50", Dubna, Russia, Apr. 21-24, 1999 (World Scientific, Singapore, 2000), p. 105; A. Kelic, I. M. Itkis, I. V. Pokrovsky, E. V. Prokhorova, B. Benoit, G. Costa, L. Donadille, O. Dorvaux, E. de Goes Brennand, G. Guillaume, F. Hanappe, B. Heusch, A. Huck, M. G. Itkis, S. Jokis, N. A. Kondratiev, E. M. Kozulin, Yu. Ts. Oganessian, G. Rudolf, A. Ya. Rusanov, L. Stuttge, D. Vorkapic, and K. Yuasa-Nakagawa, Europhys. Lett. 47(5), 552 (1999).

(24) Yu. Ts. Oganessian, V. K. Utyonkov, Yu. V. Lobanov, F. Sh. Abdullin, A. N. Polyakov, I. V. Shirokovsky, Yu. S. Tsyganov, G. G. Gulbekian, S. L. Bogomolov, B. N. Gikal, A. N. Mezentsev, S. Iliev, V. G. Subbotin, A. M. Sukhov, G. V. Buklanov, K. Subotic, M. G. Itkis, K. J. Moody, J. F. Wild, N. J. Stoyer, M. A. Stoyer, and R. W. Lougheed, Phys. Rev. Lett. 83, 3154 (1999). 